\title{
Analytical Study of Severe and Great Geomagnetic Storms
}

\author{
Amita Raizada $^{1^{*}}$ \\ ${ }^{1}$ Department of Physics, DAV PG College, Dehradun (UK), India \\ *Corresponding Author Email id: amitaraizada1971@gmail.com \\ Received: 2.8.2021; Revised: 4.9.2021; Accepted: 18.9.2021 \\ CSociety for Himalayan Action Research and Development
}

\begin{abstract}
Severe and Great Geomagnetic storms are considered to be those which have Dst less than -200nT. The investigation years are January 1996 to December 2006, which refers to $23^{\text {rd }}$ solar cycle. During this period, 18 Geomagnetic storms are observed. When there are maximum number of sunspots present at the solar disk, highest number of these Geomagnetic storms are observed, which is quite obvious. The Coronal Mass Ejections responsible for these storms depict latitudinal as well as longitudinal bias showing more inclination towards northern hemisphere and western region of the solar disk. These Coronal Mass Ejections are confined between $\pm 25^{\circ}$ of the equator.
\end{abstract}

Keywords: Sunspot number • Disturbance-time-storm index, $\bullet$ Magnetosphere

\section{Introduction}

Geosphere is greatly affected by different solar features like Solar Flares (SFs), Coronal Mass Ejections (CMEs), etc. The main cause of highly energetic geomagnetic storms (GMSs) is believed to be large interplanetary magnetic field (IMF) structure (Tsurutani et al., 1988; Echer et al., 2004; Kumar and Raizada, 2008) which interact with the Earth's magnetic field and facilitate the transport of energy into the Earth's atmosphere through the reconnection process. Solar wind (SW) and CMEs occupy the forefront of solar terrestrial research because they have been identified as the primary sources of space weather (Gopalswamy, 2011).

CMEs have been identified as the most energetic events in the heliosphere which are responsible for affecting solar wind in the form of either magnetic field disturbances or speed of solar wind or its density, and hence magnetic disturbance occurs at Earth (Zhang et al., 2003). CMEs, when evolved from Sun, move in all directions. But those, which are directed towards Earth (called geoeffective CMEs) are mainly responsible for impacting the magnetosphere and hence Geomagnetostorms (GMSs) take place (Gopalswamy, 2006; Kumar and Raizada, 2010; Manoharan, 2012). CMEs are classified as Halo, partial halo and narrow depending upon their width. Ones with apparent width of $360^{\circ}$ are termed as 'Halo', those with width less than $120^{\circ}$ are narrow, whereas CMEs with width between $120^{\circ}$ and $359^{\circ}$ are said to be 'Partial Halo'. Since Halo CMEs are wider than other CMEs, they are expected to possess more energy (Zhao, 2004; Gopalswamy, 2007). Depending upon the speed of CME, at the time of its evolution at the solar disk, it's time to reach http://jmr.sharadpauri.org 
Earth is decided, which could be anything between 24 hours to 5 days (Zhang et al., 2003). In this research paper, an attempt is made to identify the solar features and also analyze their characteristics for causing severe/ great GMSs, on the basis of disturbance storm time (Dst) index. Here, severe GMSs will be designated with $-200 \mathrm{nT}>$ Dst $\geq-350 \mathrm{nT}$ and great GMSs with Dst $<-350 \mathrm{nT}$, as given by Loewe and Prolss in 1997.

\section{Data analysis}

The period chosen here is from January 1996 to December 2006 which covers $23^{\text {rd }}$ solar cycle. During these years, 18 Geomagnetic storms falling under the category of Severe and Great are observed. There are some gaps present in the available data, may be due to weather condition or servicing/maintenance of instruments etc. Due to which, two GMSs of September 24, 1998 and October 21, 1999 are not investigated thoroughly. The data used here is freely available at internet so as to facilitate different researchers across the globe and is collected by different research centers, which are acknowledged by the author in 'References'

\section{Results and discussion}

Different number of sunspots appears at solar disk at different time which goes on increasing from minimum to maximum and then declines back to minimum in 11 years time. Somehow, $23^{\text {rd }}$ solar cycle extended till 12.3 years and marks the period from 1996 to 2008. 18 GMSs have occurred during the investigation period. Out of which 14 are of severe and 4 are of Great nature. The value of Dst, which is the indicator of commencement of GMS, starts falling many hours before the storm and attains the minimum value when the storm is at its maximum strength.

It has been observed that the frequency of CMEs varies with sunspot cycle. Lesser number of sunspots indicates less activity at solar disk and hence lesser number of CMEs evolves. The number of CMEs increases as the solar cycle progresses. Approximately 2 to $3 \mathrm{CMEs} /$ day are observed when solar cycle is at its peak. Thus, large number of GMSs with Dst $<-200 n T$ are expected close to the solar maximum, which is clear from Fig. 1. Ten GMSs of severe and great nature have been observed during maximum phase (1999 to 2002), while only 2 and 6 GMSs showed up in rising (1996 to 1998) and declining (2003 to 2006) periods respectively. The mean value of the speed of solar wind velocity (Vsw) for the storms under investigation is $614 \mathrm{Km} / \mathrm{s}$ with minimum speed of $362 \mathrm{Km} / \mathrm{s}$ and maximum of $866 \mathrm{Km} / \mathrm{s}$. The minimum value attained by Dst index for a particular GMSs against Solar wind velocity (Vsw), has been plotted in Fig. 2. 


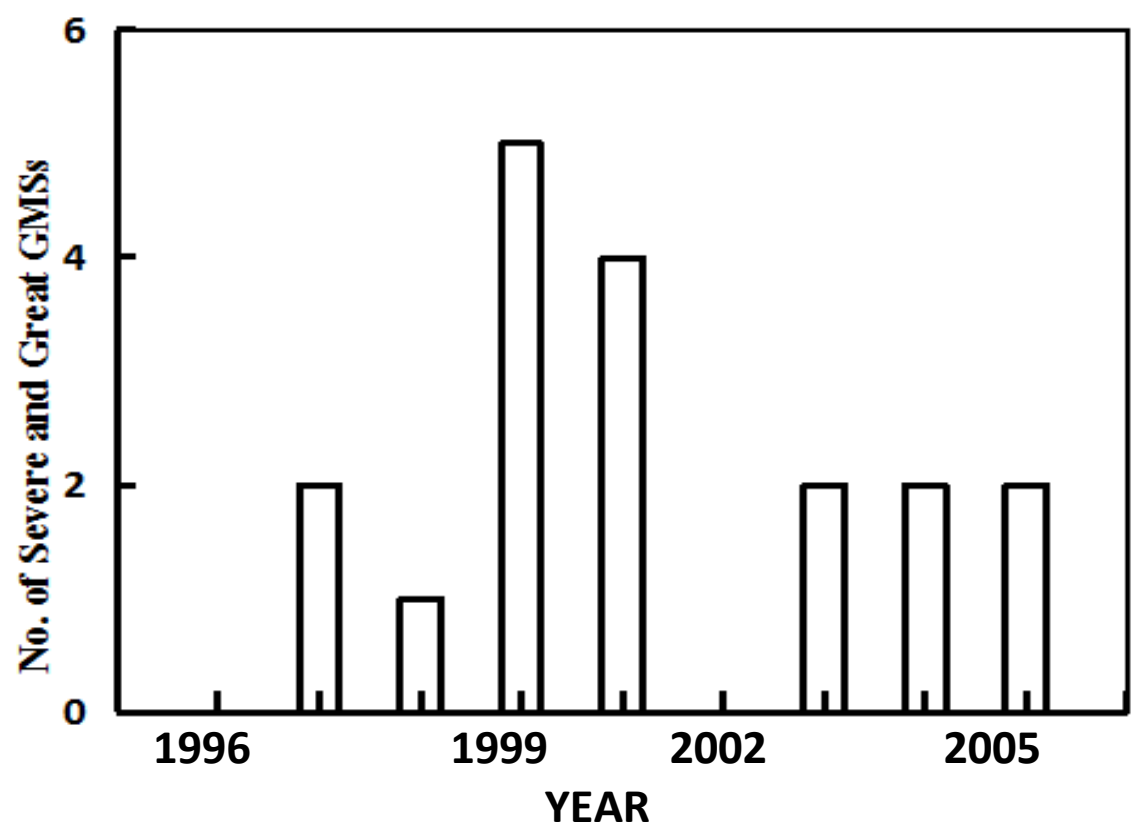

Fig.1 Yearly variation of Severe and Great GMSs during Solar cycle 23

The CME accelerates or decelerates during its propagation from solar disk towards Earth, in comparison to its speed at the time of its evolution, which is termed as initial speed. The minimum initial speed of CMEs due to which these GMSs occur, is found to be $453 \mathrm{Km} / \mathrm{s}$ and maximum is $2459 \mathrm{Km} / \mathrm{s}$ respectively with an average value of $1682 \mathrm{Km} / \mathrm{s}$ which is pretty high. Fig. 3 shows the minimum value attained by Dst index against initial speed of CME.

The investigation reveals that CMEs are responsible for all the events (severe and great both) to take place. It could be possible that more than one CME is involved in the process. Thorough investigation shows that Active Prominences and Disappearing Filaments, which is one of the many solar features, are also associated with $23 \%$ events. Besides this, all the events are associated with SFs of $\mathrm{M}$ and $\mathrm{X}$ class i.e. of high importance. SOHO observations support that the flare and CME are two manifestations of the same energy release (Gopalswamy, 2016).

The locations of CMEs for these severe and great GMSs have been plotted in Fig. 4 which clearly depicts that the location of these geoeffective CMEs is not symmetrical over the solar surface. $37 \%$ evolve from eastern side while $63 \%$ from western side. Thus, longitudinal distribution shows more inclination towards west side. Further, $81 \%$ of the total events observed are the disk events where the longitude 
is confined within $\pm 45^{\circ}$ of the central meridian and not the limb events where the longitude is confined between $45^{\circ}$ and $90^{\circ}$ which is similar to the findings of Gopalswamy et al., 2007. Fig. 4 also suggests the hemispherical bias in location of these CMEs. $69 \%$ of these CMEs are

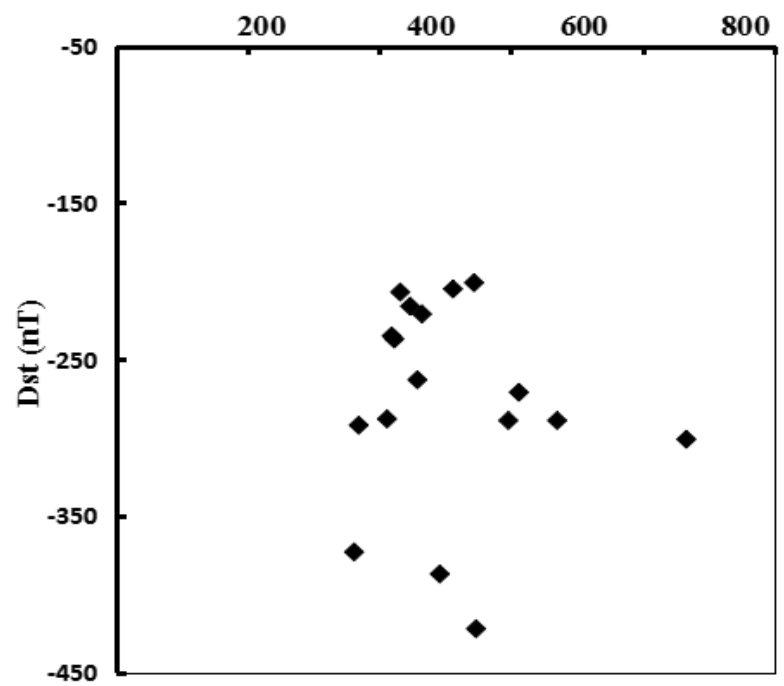

Fig 2: Dst (minimum) value versus Solar Wind speed

\section{Conclusions}

The analytical study reveals that the largest number of severe and great GMSs happen during maximum phase of solar cycle. The high speed Solar Wind Plasma, could be responsible for these severe/ great Geomagnetic storms. These GMSs are mainly associated with halo CMEs followed by SFs of high importance. The geoeffective CMEs causing severe and great GMSs show longitudinal as well as observed to have occurred in north part and $31 \%$ in south part of solar disk. However, looking at the latitudinal position from the equator, the geoeffective CMEs are found in the vicinity of $\pm 25^{\circ}$ from the position of equator.

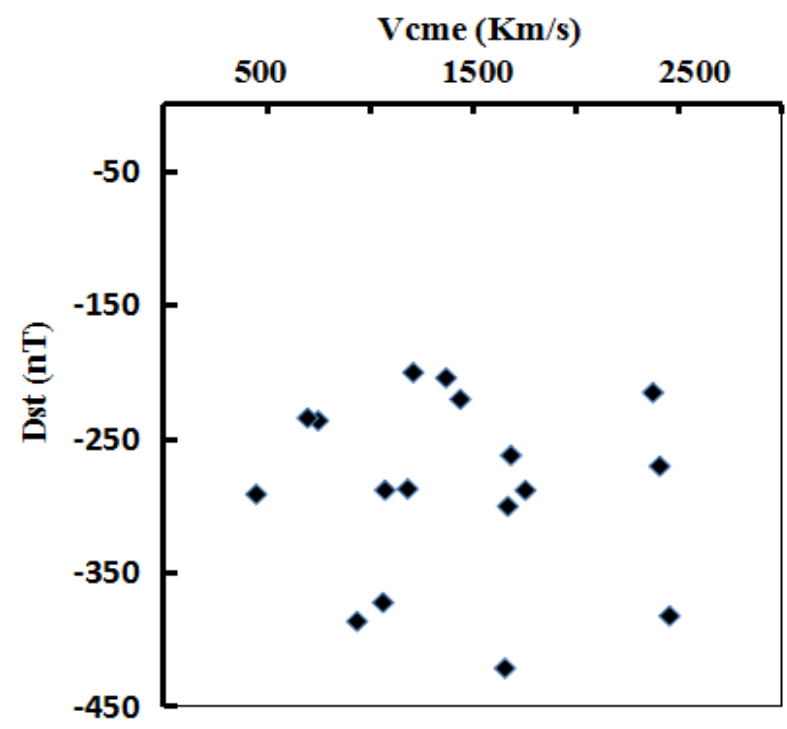

Fig. 3 Dst (minimum) versus CME speed.

hemispherical bias. These CMEs are generally confined close to the equator and are mainly the disk events. The correlation coefficient has not been calculated for Vsw against Dst as well as for Vcme against Dst because the data sample is too small for statistical analysis. The study can be extended with considerable data sample so as to find solar and interplanetary parameters for predicting these storms. 


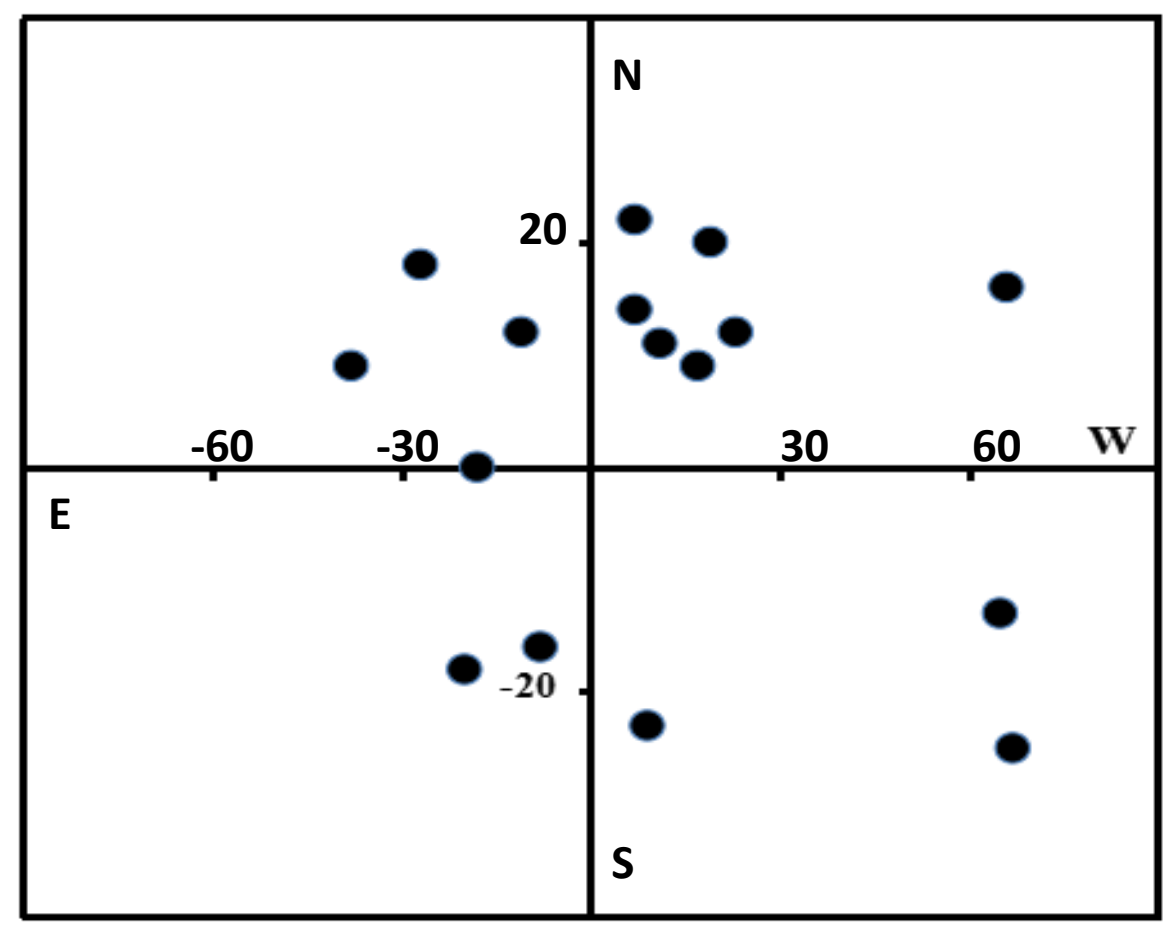

Fig. 4 Geoeffective CMEs' location on Solar disk.

\section{References}

Echer E, Alves MV and Gonzalez WD (2004) Geoeffectiveness of interplanetary shocks during solar minimum (1995-1996) and solar maximum (2000). Solar Phys. 221: 361-380

Gopalswamy N (2006) Coronal mass ejections of solar cycle 23. J. Astrophys. \& Astron. 27:243- 254 referen(and ces there in)

Gopalswamy N, Yashiro S and Akiyama S (2007) Geoeffectiveness of halo coronal mass ejection. J. Geophys. Res. 112: A06112 (and references there in)

Gopalswamy N (2011) Universal heliophysical processes.Chapter in The Sun, the solar wind and the heliosphere, DOI 10.1007/978-90-481-9787-3 2: 9-20

Gopalswamy N (2016) History and development of coronal mass ejections as a key player in terrestrial relationship. Geosci. Lett. 3: 8: DOI 10.1186/s40562-016-0039-2
Kumar S and Raizada A(2008) Effect of solar features and interplanetary parameters on geomagnetosphere during solar cycle 23. Pramana J. Phys. 71: 1353-1366

Kumar S and Raizada A(2010) Geoeffectiveness of magnetic clouds occurred during solar cycle 23. Planetary and space science 58:741-748

Loewe CA and Prolss GW (1997) Classification and mean behaviourof magnetic storms. J. Geophys. Res. 102: 14209-14213

Manoharan PK (2012) Three dimensional evolution of solar wind during solar cycles 22 - 24. Astrophys. J. 751: 128-141

Tsurutani BT Gonzalez WD, Tang F, Akasofu SI and Smith EJ (1988) Origin of interplanetary southward magnetic fields responsible for major magnetic storms near solar maximum. (1978-1979). J. Geophys. Res. 93: 8519-8531 
Web Data sites: World data Center, Japan; SOHO/LASCO CME catalogue; OMNIWEB data; Solar Geophysical data.

Zhao XP (2004) Identification of earth directed partial halo coronal mass ejections. Int. Astronomical Union Symp. Russia 223: 569-572

Zhang J Dere KP, Howard RA and Bothmer V (2003) Identification of solar sources of major geomagnetic storms between 1996 and 2000. The Astrophys. Journal 582: 520 $-533$ 